\title{
Plasma formation and relaxation dynamics in fused silica driven by femtosecond short- wavelength infrared laser pulses
}

Cite as: Appl. Phys. Lett. 115, 191903 (2019); https://doi.org/10.1063/1.5117837

Submitted: 01 July 2019 . Accepted: 21 October 2019 . Published Online: 05 November 2019

P. Jürgens (D) M. J. J. Vrakking (D), A. Husakou (D), R. Stoian (D), and A. Mermillod-Blondin (D)

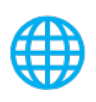

\section{ARTICLES YOU MAY BE INTERESTED IN}

Spatially resolved optical excitation of mechanical modes in graphene NEMS

Applied Physics Letters 115, 193102 (2019); https://doi.org/10.1063/1.5111755

Threshold photodissociation dynamics of $\mathrm{NO}_{2}$ studied by time-resolved cold target recoil ion momentum spectroscopy

The Journal of Chemical Physics 151, 174301 (2019); https://doi.org/10.1063/1.5095430

Thermal phase fluctuations in optically pumped dual-frequency vertical external-cavity surface-emitting lasers for cesium clocks based on coherent population trapping

Journal of Applied Physics 126, 173104 (2019); https://doi.org/10.1063/1.5123444

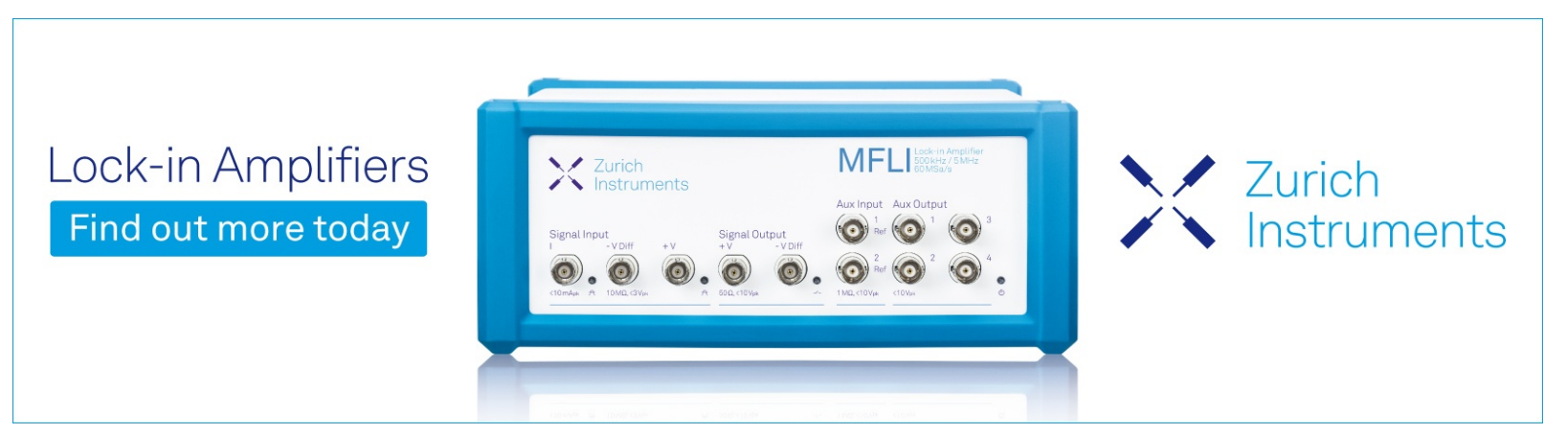




\title{
Plasma formation and relaxation dynamics in fused silica driven by femtosecond short-wavelength infrared laser pulses
}

Cite as: Appl. Phys. Lett. 115, 191903 (2019); doi: 10.1063/1.5117837

Submitted: 1 July 2019 - Accepted: 21 October 2019 .

Published Online: 5 November 2019

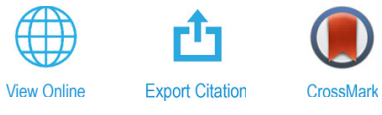

P. Jürgens, ${ }^{1, a)}$ (D M. J. J. Vrakking, ${ }^{1}$ (D) A. Husakou, ${ }^{1}$ iD R. Stoian, ${ }^{2}$ (D) and A. Mermillod-Blondin ${ }^{1}$

\author{
AFFILIATIONS \\ ${ }^{7}$ Max-Born-Institute for Nonlinear Optics and Short Pulse Spectroscopy, Max-Born-Str. 2A, D-12489 Berlin, Germany \\ ${ }^{2}$ Laboratoire Hubert Curien, UMR CNRS 5516, Unversité de Lyon, Unversité Jean Monnet, 42000 Saint Etienne, France \\ a)Electronic mail: juergens@mbi-berlin.de
}

\begin{abstract}
Laser-induced plasma formation and subsequent relaxation in dielectric solids is the precursor to structural modifications serving as the basis for direct laser writing of functional optical micro- and nanostructures. Based on an experimental arrangement combining a timeresolved transmission measurement with a cross-phase modulation measurement, we isolate the plasma formation and relaxation dynamics in the bulk of amorphous fused silica excited by femtosecond short-wavelength infrared $(\lambda=2100 \mathrm{~nm})$ laser pulses. Whereas the relaxation time of the generated subcritical electron-hole plasma was so far assumed to be constant, our findings indicate an intensity-dependent relaxation time. We attribute this intensity dependence to vibrational activation of the medium, leading to detrapping of trapped carriers and a reduced trapping probability.
\end{abstract}

Published under license by AIP Publishing. https://doi.org/10.1063/1.5117837

Studying ultrafast optical breakdown in solid dielectrics is of paramount importance to understand, control, and optimize laser-induced micro/nanoprocessing of transparent materials. ${ }^{1,2}$ Ultrafast optical breakdown relies on the injection of initially bound electrons from the valence band into the conduction band. An initial population of quasifree electron-hole pairs is generated by laser-induced strong-field ionization (SFI). The electron-hole plasma is subsequently heated through inverse bremsstrahlung (free carrier absorption), resulting in multiplication of the free carrier density via electron-impact ionization (IMP). ${ }^{3}$

The dynamics of the plasma decay provide microscopic insights into the underlying relaxation pathways, whereby the kinetics of the relaxation serve as a marker for the electronically and thermally induced structural reorganization of the matrix. The decay of the conduction band electron density might result in the emission of (incoherent) light by direct radiative recombination or-more importantly for this letter-in the conversion of electronic energy into lattice energy by electron-phonon coupling and defect formation. In strongly coupled dielectrics such as fused silica, the formation of defects like nonbridging oxygen-hole centers and $\mathrm{E}^{\prime}$ centers is preceded by ultrafast self-trapping of quasifree carriers. ${ }^{8}$ The self-trapping is related to the matrix polarizability and the ability of the carrier to influence the molecular dipole. This interaction can be visualized as a deformation potential induced by the carrier itself in opposition to existing traps or distortions.

To investigate the dynamics of the ultrafast relaxation processes, time-resolved studies of excitation and relaxation dynamics have been carried out extensively in the past few decades. ${ }^{5,10-18}$ In fused silica, the formation of self-trapped excitons (STEs) has been identified as the major source for ultrafast plasma relaxation, ${ }^{10,11}$ which has been confirmed by resonantly probing the STE absorption bands in the ultraviolet (at 220 and $240 \mathrm{~nm}$ ) spectral domain ${ }^{19,20}$ (see the inset in Fig. 1 for a sketch of the contributing energy levels). As a result of these experimental studies, constant decay times ranging from 50 to $220 \mathrm{fs}$ (see Table I) were reported and used as such in numerical simulations where the plasma relaxation rate is usually modeled by a single intensity-independent exponential decay term associated with the formation of self-trapped excitons. ${ }^{5,21,22}$

In this Letter, we measure the decay rate of the plasma transmission (i.e., $\tau_{\text {rel }}$ ) vs the excitation intensity using time-resolved transient absorption. Our results show a monotonous increase in $\tau_{\text {rel }}$ as the intensity increases. We attribute this increase to vibrational activation of the irradiated target due to the collisions of the quasifree electrons with the lattice. Our interpretation is supported by numerical simulations of the energy coupled from the laser field into the material and of 


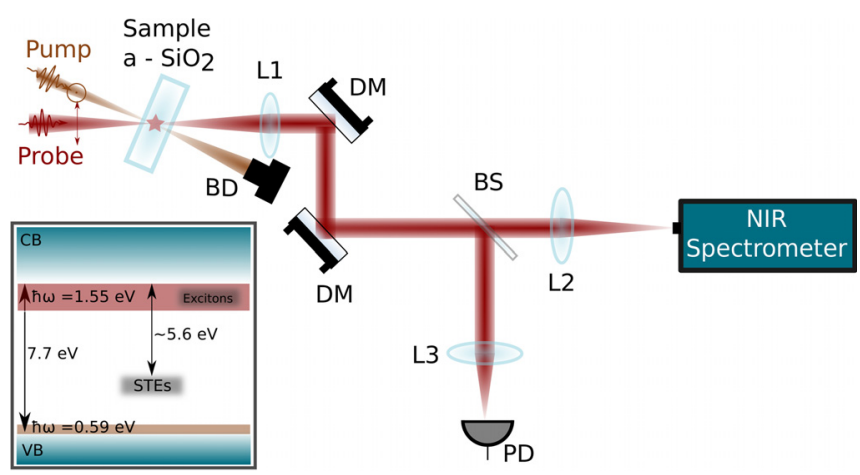

FIG. 1. Experimental setup for the cross-polarized, close-to-collinear pump-probe investigations. L1-L3: lenses, BD: beam dump, DM: dielectric mirrors (HR $800 \mathrm{~nm}$ ), BS: beam splitter, PD: photodiode, and NIR spectrometer: near-infrared spectrometer. The inset shows an energy diagram of the valence and conduction bands separated by an energy difference of $7.7 \mathrm{eV}$ together with the laser-induced interband levels of the excitons and the self-trapped excitons. The photon energies of the pump and the probe laser pulse are visualized in the vicinity of the valence band and the conduction band, respectively.

the relative contribution of SFI and IMP to the temporal evolution of the conduction band electron density.

The experimental setup [see Fig. 1(b)] is based on a commercial Ti:sapphire laser system (Spectra Physics Spitfire Pro) delivering linearly polarized laser pulses of $\approx 40 \mathrm{fs}$ full width half maximum (FWHM) duration at $1 \mathrm{kHz}$ repetition rate around a central wavelength of $794 \mathrm{~nm}$. A fraction of the pulses $(\approx 1.1 \mathrm{~mJ})$ is used to pump a commercial optical parametric amplifier (Light Conversion TOPAS C) producing an idler at a central wavelength of $2100 \mathrm{~nm}$ with up to $50 \mu \mathrm{J}$ energy per pulse and a FWHM duration of $\approx 140 \mathrm{fs}$. The idler is employed as an excitation pulse in the experiment, while a small fraction of the remaining near-infrared (NIR) laser pulse is utilized as a weak probe pulse. Both pulses are temporally and spatially overlapped inside a fused silica sample of $500 \mu \mathrm{m}$ thickness (UV grade fused silica Corning7980, $E_{\text {gap }} \approx 7.7 \mathrm{eV}$ ) using a cross-polarized, close-to-collinear $\left(\alpha_{\text {exc-pr }}<5^{\circ}\right)$ pump probe geometry. Due to the quasicollinear arrangement of the beams and the much shorter probe pulse duration compared to the duration of the pump laser pulse, no significant influence of the pump-probe angle on the temporal resolution is expected. ${ }^{23}$ The SWIR pump laser pulse is focused using a gold-coated off-axis parabolic mirror (OAP) with a focal length of $50 \mathrm{~mm}$, inducing a focal spot size of

TABLE I. Overview of experimentally determined relaxation times $\left(\tau_{\text {rel }}\right)$ in fused silica from pump-probe measurements.

\begin{tabular}{|c|c|c|}
\hline References & Exc. Wavelength & $\tau_{\text {rel }}$ \\
\hline Audebert et al. ${ }^{10}$ & $620 \mathrm{~nm}$ & $150 \mathrm{fs}$ \\
\hline Martin et al. ${ }^{11}$ & $395 \mathrm{~nm}$ & $150 \mathrm{fs}$ \\
\hline Li et al. ${ }^{5}$ & $800 \mathrm{~nm}$ & $60 \mathrm{fs}$ \\
\hline Mero et al. ${ }^{12}$ & $800 \mathrm{~nm}$ & $220 \mathrm{fs}$ \\
\hline Sun et al. ${ }^{13}$ & $800 \mathrm{~nm}$ & $170 \mathrm{fs}$ \\
\hline Grojo et al. ${ }^{14}$ & $800 \mathrm{~nm}$ & $179 \mathrm{fs}$ \\
\hline Pan et al. ${ }^{18}$ & $800 \mathrm{~nm}$ & $50 \mathrm{fs}$ \\
\hline
\end{tabular}

$33 \mu \mathrm{m}$ (radius at $I_{0} / e^{2}$, measured with a knife edge technique in air), while the weak NIR probe laser pulse is focused by a silver coated focusing mirror (focal length: $300 \mathrm{~mm}$ ) through a central hole in the OAP, down to a spot size of $75 \mu \mathrm{m}$. The fact that the probe beam is larger than the focal spot of the pump laser beam only affects the absolute values that are recorded, whereas the observed dynamics remain unaffected. After recollimation, the weak probe laser pulse is split into two equally intense parts. One part is directed toward a photodiode (Ophir PD-10) in order to characterize the transmission of the time-delayed probe laser pulse following the modification of the target by the pump laser, while the second part is spectrally analyzed using a NIRspectrometer (Avantes Avaspec-LS2048).

The fused silica sample is mounted on a computer-controlled $x-y$ translation stage enabling scanning of the target during irradiation in order to provide a fresh spot for every laser shot. A motorized linear translation stage inserted into the beam path of the probe laser pulse allows the acquisition of transmission data and probe spectra as a function of the pump-probe delay $\tau$. Figure 2(a) displays the detected time-resolved probe spectrum at an incident SWIR intensity of $11 \mathrm{TW}$ $\mathrm{cm}^{-2}$. Cross-phase modulation (XPM) between the strong SWIR pump laser pulse and the weak NIR probe laser pulse results in strong distortions of the probe spectrum during pump-probe overlap and is used to calibrate the zero of the time delay between the pump and the probe in the experiment. From the time-frequency map shown in Fig. 2(a), we extract the evolution of the central probe wavelength by calculating the center of gravity of the spectrum at each delay. The resulting variation of the central probe wavelength as a function of the pumpprobe delay is shown in Fig. 2(b). A strong redshift at negative delays is followed by an equally large blueshift at positive pump-probe delays. During its propagation through the pump-affected region in the fused silica sample, the probe pulse reads out refractive index variations
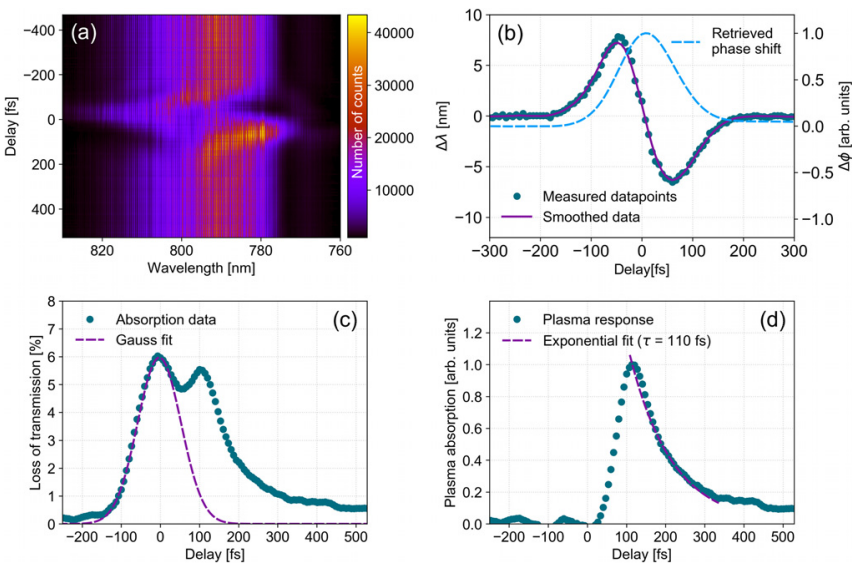

FIG. 2. Experimental results obtained at a pump laser intensity of $11 \mathrm{TW} \mathrm{cm}^{-2}$. (a) Time-frequency map of the probe spectrum as a function of the delay between the pump and the probe laser pulse, revealing a delay-dependent shift of the probe spectrum due to cross-phase modulation (XPM). (b) Extracted dynamics of the central probe wavelength together with the phase shift induced by the SWIR pump laser pulse in the fused silica target retrieved from direct integration of the measured XPM signal. (c) and (d) Experimental absorption measurements obtained using the photodiode. (c) Total loss of transmission as a function of the delay between the pump and the probe laser pulse. (d) Isolated plasma dynamics with an exponential fit to the decay of the absorption profile. 
induced by the strong excitation pulse. As a result, the central probe wavelength varies according to (compare, e.g., Ref. 24)

$$
\begin{aligned}
\Delta \omega(\tau) & =\frac{\int_{-\infty}^{+\infty} I_{\text {probe }}(t) \frac{\partial \phi(t, \tau)}{\partial t} d t}{\int_{-\infty}^{+\infty} I_{\text {probe }}(t) d t} \\
& \approx-\left.\frac{2 \pi L}{\lambda_{0}} \frac{\partial n\left(I_{\text {pump }}(t)\right)}{\partial t}\right|_{\tau},
\end{aligned}
$$

with $\tau_{\text {pump }}>\tau_{\text {probe. }}$. Here, $L$ defines the interaction length, $\lambda_{0}$ is the initial central probe wavelength, and $n$ denotes the refractive index of the sample, which is related to the pump laser intensity $I_{\text {pump }}(t, \tau)$ via the optical Kerr-effect. $I_{\text {probe }}(t)$ is the probe laser intensity and as before $\tau$ denotes the pump-probe delay. Thus, the integrated variation of the central probe wavelength reveals the delay dependence of the phase shift $\Delta \phi(\tau)$ induced by the intense SWIR laser pulse [see Fig. 2(b)]. In what follows, the pump-probe zero delay $(\tau=0)$ is arbitrarily defined as the delay providing the maximum of $\Delta \phi$.

The measured time-resolved loss of transmission of the probe laser pulse through the region affected by the pump is presented in Fig. 2(c). A double-peaked structure is observed with a first peak appearing at the pump-probe overlap followed by a second peak delayed by $\approx 100 \mathrm{fs}$. After the second peak, the absorption slowly decays toward its initial value. We attribute the first peak to a nonlinear interaction of the pump and probe laser pulses, which leads to a reversible depletion of the probe laser pulse recently described as a two-beam coupling process. ${ }^{17}$ We assign the second peak to absorption in the generated electron-hole plasma. We emphasize that the direct absorption by STEs can be excluded as a major source of our absorption signal since their absorption bands lie far away from the photon energy of the probe laser used in our experiments (see the inset in Fig. 1). Considering the field strengths used in this study, intensity clamping limits the amount of bulk excitation to subcritical densities. ${ }^{25,26}$ Therefore, the loss of transmission due to an increased reflectivity of the created electron-hole plasma can be neglected. ${ }^{15,27}$ In order to isolate the plasma response from the reversible two-beam coupling effects, we apply a Gaussian fit to the leading edge of the first peak and subtract it from the total absorption curve to obtain the isolated plasma absorption as shown in Fig. 2(d). From the displayed dynamics of the probe transmission in Figs. 2(c) and 2(d), it is obvious that ultrafast carrier trapping plays a predominant role in the decay of the generated electron-hole plasma. Therefore, we approximate the relaxation of quasifree carriers from the conduction band by a single exponential decay time $\tau_{\text {rel }}$ as suggested in, e.g., Refs. 11 and 14, leading to the determination of a relaxation time of $\tau_{\text {rel }}=110 \mathrm{fs}$.

In order to further analyze the relaxation dynamics of the generated electron-hole plasma, we perform the time-resolved XPM and transmission measurements as a function of the SWIR excitation intensity. We vary the intensity of the pump laser pulse up to values slightly above the single-shot threshold for irreversible modification that is identified by the detection of a scattering signal from the sample and additional postmortem microscope investigations of the induced surface modifications. As the maximum intensity only slightly exceeds the material modification threshold, our measurement is only sensitive to plasma dynamics within the bulk of the fused silica sample in contrast to recent experiments, demonstrating the emergence of different relaxation phenomena in a thin ablating layer on the surface. ${ }^{15}$ The results obtained for the maximum value of the plasma absorption and the fitted relaxation time as a function of the excitation strength are summarized in Figs. 3(a) and 3(b). Due to the highly nonlinear excitation process, the maximum value of the absorption associated with the formation of the electron-hole plasma monotonously increases as a function of excitation intensity in a nonlinear fashion [see Fig. 3(a)]. Surprisingly, the fitted relaxation time displays a similar behavior. While only intensity-independent relaxation times have been observed in $\mathrm{SiO}_{2}$ until now, ${ }^{11}$ we detect an extremely fast relaxation with a characteristic time of $\approx 50 \mathrm{fs}$ at intensities far below the damage threshold. Once the intensity of the SWIR excitation pulse approaches the threshold for irreversible modification of the fused silica target [see colored areas in Figs. 3(a) and 3(b)], the relaxation time increases. Still, a further increase in the excitation intensity to values clearly above the damage threshold leads to the observation of even longer decay times.

We attribute the observation of an intensity-dependent relaxation time to vibrational activation of the material due to the energy stored in the free carrier bath. Once this energy is transferred to the lattice, it induces modifications of the deformation potential, leading to both a decreased ability of the carrier to be trapped and an increased chance for already trapped carriers to be detrapped. As shown by many studies, thermal detrapping occurs in various materials (see, e.g., Refs. 28 and 29 and references therein). Here, energy is coupled from the electron-hole plasma to the lattice by electron-phonon coupling, leading to vibrations of the lattice. Once the vibrational energy is sufficient, a STE can be converted back into a quasifree electron hole pair, while at the same time the trapping probability is reduced, resulting in an effectively prolonged relaxation time of the laser-induced electronhole plasma. As these two mechanisms are correlated effects that result from the same process, they are expected to be experimentally
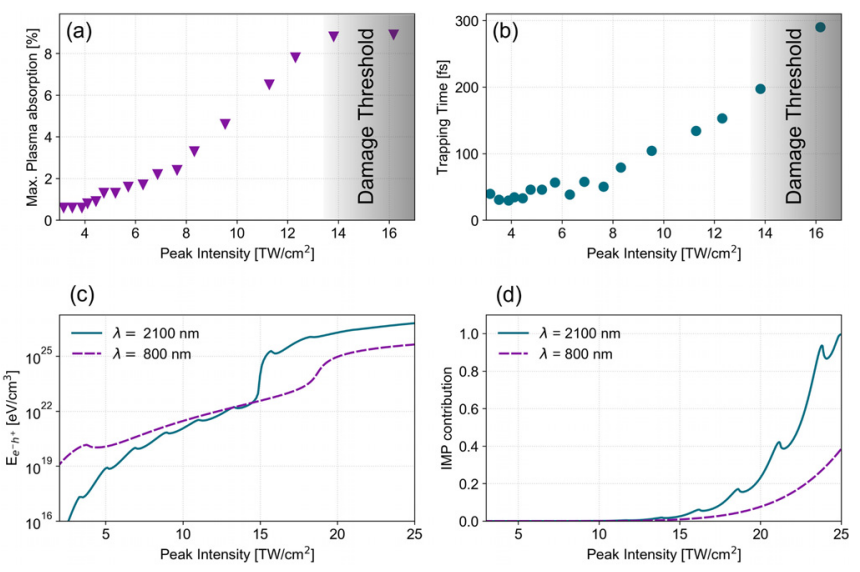

FIG. 3. (a) and (b) Experimental determination of the maximum absorption strength and the relaxation times obtained from the pump intensity scans. (a) Maximum value of the retrieved plasma absorption as a function of the excitation strength. (b) Intensity dependence of the relaxation times obtained by applying exponential fits to the decay of the isolated plasma absorption profiles. (c) Numerical simulation according to Eq. (2) of the energy coupled into the free carrier bath as a function of the laser intensity for a central wavelength of 2100 and $800 \mathrm{~nm}$. (d) Numerical results for the relative contribution of IMP[AQ] with respect to SFI obtained by solving Eq. (3). The pulse duration in (c) and (d) corresponds to 20 optical cycles of the driving field. 
indistinguishable. Because heating of the free carrier bath mainly proceeds through inverse bremsstrahlung, ${ }^{30,31}$ the described detrapping mechanism is particularly pronounced at long laser wavelengths. Since previous experiments were mainly performed using near-infrared laser pulses (see Table I) where the intensity dependence of the relaxation time is expected to be much weaker, only constant relaxation times for the electron-hole plasma in fused silica have been observed.

Irrespective of the wavelength, the energy stored in the electronhole plasma can be estimated by using the condition for energy conservation

$$
E_{\mathrm{e}^{-} \mathrm{h}^{+}} \approx \int_{-\infty}^{+\infty} \rho(I, t) \times V \times\left[E_{\text {gap }}+U_{\mathrm{p}}(I, t)\right] d t,
$$

with plasma density $\rho(I)$, volume $V$, bandgap energy $E_{\text {gap }}$, and ponderomotive energy $U_{\mathrm{p}}$. The ponderomotive energy, i.e., the cycleaveraged electron quiver energy is defined through $U_{p}=e^{2} E_{0}^{2} /$ $\left(4 \pi m^{*} \omega^{2}\right)$ (we neglect the kinetic energy of the holes due to their comparably high mass). Here, $e$ is the elementary charge, $E_{0}$ is the amplitude of the laser electric field, $m^{*}$ denotes the reduced electron mass in the conduction band $\left(m^{*}=0.635 m_{e}\right.$ for fused silica $\left.{ }^{30}\right)$, and $\omega$ is the central laser frequency of the driving field. Equation (2) can be evaluated with the help of a single rate equation for the temporal evolution of the conduction band electron density given by ${ }^{32-34}$

$$
\frac{\partial \rho(t)}{\partial t}=\Gamma_{\mathrm{SFI}}+\Gamma_{\mathrm{IMP}}-\frac{\rho(t)}{\tau_{\text {rel }}} .
$$

Here, $\Gamma_{\mathrm{SFI}}$ denotes the generation of quasifree carriers by strong-field ionization, $\Gamma_{\text {IMP }}$ describes the variation of the conduction band electron density induced by electron-impact ionization, and the relaxation of quasifree carriers from the conduction band is approximated by a single exponential decay time $\tau_{\text {rel }}$.

Numerical solutions of Eq. (2) as a function of the pump laser intensity are presented in Fig. 3(c) using the strong-field ionization rate provided by Keldysh, ${ }^{35}$ a modified Drude model for electronimpact ionization as presented in Ref. 21 and common material parameters for fused silica $\left(E_{g}=7.5 \mathrm{eV}, \tau_{\text {rel }}=220 \mathrm{fs}\right)$. The calculations are carried out for a NIR and a SWIR pump laser wavelength and a pulse duration corresponding to 20 optical cycles (reproducing our experimental conditions). Clearly, the energy coupled into the electron-hole plasma is much larger in the $2100 \mathrm{~nm}$ case above a certain threshold intensity $\left[\approx 15 \mathrm{TW} \mathrm{cm}^{-2}\right.$ in Fig. 3(c)]. Comparing this observation to the simulation of the relative contribution of IMP to the total generated conduction band electron density [see Fig. 3(d)] indicates that this threshold intensity corresponds to the onset of IMP, playing a significant role in the formation of the electron-hole plasma. Since no propagation effects are included in the simulation, the intensity values in the simulation are significantly above the experimental values. Naturally, the nonlinear effects like self-focusing lead to an increase in the laser pulse intensity inside the material, which leads to a local increase in the intensity, resulting in a decrease in the laser pulse energy required to initiate plasma formation. ${ }^{36}$

The differences between the NIR and SWIR scenarios found in the simulations illustrate that the signature of vibrational activation is more pronounced in the case of a long wavelength driving field due to the higher average kinetic energy of the excited electrons. The fact that IMP plays a major role in the formation of the observed electron-hole plasma is furthermore supported by the delay of the maximum plasma density with respect to the overlap of the pump and probe pulse [see Figs. 2(a) and 2(b)]. Hence, the observation of an intensity-dependent relaxation time requires sufficient vibrational energy coupled into the lattice to influence carrier trapping. Additionally, a significant contribution of IMP to the total generated density of quasifree carriers further assists the vibrational activation mechanism.

In conclusion, time-resolved transmission and XPM measurements have been performed on amorphous fused silica samples excited by femtosecond SWIR laser pulses. A strong dependence of the relaxation time of excited conduction band electrons on the present electric field strength was observed. We attribute this dependence to strongly increased energy coupling into the free carrier bath at the SWIR pump wavelength used in the experiment. Enhancing the energy deposition in the free carrier bath results in a vibrational activation of the medium with two important consequences. First, the vibrational activation results in detrapping of self-trapped charges and second, it weakens the self-trapping efficiency by altering the deformation potential. We emphasize that such an interpretation is compatible with the observation of quasiconstant relaxation times at shorter wavelengths (especially in the UV and VIS domains), where the ponderomotive energy and the influence of IMP are not sufficient to significantly modify the carrier trapping probability.

Our results extend the present literature on time-resolved studies of laser-matter interaction into the SWIR spectral domain and provide a method to isolate the persistent plasma response from transient, reversible effects based on the simultaneous detection of an XPM signal in bulk solids. The observation of the influence of lattice vibrations on the trapping efficiency provides a step toward an improved understanding of the fundamental mechanisms underlying laser micromachining with long wavelength driving fields. Finally, we expect a refinement of theoretical models describing ultrafast laser-matter interaction based on the implementation of intensity-dependent relaxation phenomena.

The authors would like to acknowledge the financial support of the German Research Foundation DFG (Grant No. ME 4427/1-1 \& ME 4427/1-2). We thank Dr. Marco Jupé (Laser Zentrum Hannover e. V.) for measuring the bandgap of the samples used in this study.

\section{REFERENCES}

${ }^{7}$ R. R. Gattass and E. Mazur, Nat. Photonics 2, 219 (2008).

${ }^{2}$ A. Ródenas, M. Gu, G. Corrielli, P. Paié, J. Sajeev, A. K. Kar, and R. Osellame, Nat. Photonics 13, 105 (2019).

${ }^{3}$ D. Du, X. Liu, G. Korn, J. Squier, and G. Mourou, Appl. Phys. Lett. 64, 3071 (1994).

${ }^{4}$ M. Lenzner, J. Krüger, S. Sartania, Z. Cheng, C. Spielmann, G. Mourou, W. Kautek, and F. Krausz, Phys. Rev. Lett. 80, 4076 (1998).

${ }^{5}$ M. Li, S. Menon, J. P. Nibarger, and G. N. Gibson, Phys. Rev. Lett. 82, 2394 (1999).

${ }^{6}$ S. Guizard, S. Klimentov, A. Mouskeftaras, N. Fedorov, C. Geoffroy, and G. Vilmart, Appl. Surf. Sci. 336, 206 (2015).

${ }^{7}$ E. G. Gamaly and A. V. Rode, Prog. Quantum Electron. 37, 215 (2013).

${ }^{8}$ N. Itoh, T. Shimizu-Iwayama, and T. Fujita, J. Non-Cryst. Solids 179, 194 (1994).

${ }^{9}$ R. T. Williams and K. S. Song, J. Phys. Chem. Solids 51, 679 (1990).

${ }^{10}$ P. Audebert, P. Daguzan, A. Dos Santos, J. C. Gauthier, J. Geindre, S. Guizard, G. Hamoniaux, K. Krastev, P. Martin, G. Petite, and A. Antonetti, Phys. Rev. Lett. 73, 1990 (1994). 
"P. Martin, S. Guizard, P. Daguzan, G. Petite, P. D’Oliveira, P. Meaynadier, and M. Perdrix, Phys. Rev. B 55, 5799 (1997).

${ }^{12}$ M. Mero, J. Liu, A. Sabbah, J. Jasapara, K. Starke, D. Ristau, J. K. McIver, and W. Rudolph, Proc. SPIE 4932, 202-212 (2003).

${ }^{13}$ Q. Sun, H. Jiang, Y. Liu, Z. Wu, H. Yang, and Q. Gong, Opt. Lett. 30, 320 (2005).

${ }^{14}$ D. Grojo, M. Gertsvolf, S. Lei, T. Barillot, D. M. Rayner, and P. B. Corkum, Phys. Rev. B 81, 212301 (2010).

${ }^{15}$ M. Garcia-Lechuga, L. Haahr-Lillevang, J. Siegel, P. Balling, S. Guizard, and J. Solis, Phys. Rev. B 95, 214114 (2017).

${ }^{16}$ T. Winkler, L. Haahr-Lillevang, C. Sarpe, B. Zielinski, N. Götte, A. Senftleben, P. Balling, and T. Baumert, Nat. Phys. 14, 74 (2018).

${ }^{17}$ S. Ye and M. Huang, preprint arXiv:1803.02566 [physics.optics] (2018).

${ }^{18}$ C. Pan, L. Jiang, W. Wang, J. Sun, G. Wang, and Y. Lu, Appl. Phys. Lett. 112, 191101 (2018).

${ }^{19}$ P. N. Saeta and B. I. Greene, Phys. Rev. Lett. 70, 3588 (1993).

${ }^{20}$ S. Guizard, P. Martin, G. Petite, P. D'Oliveira, and P. Meynadier, J. Phys.: Condens. Matter 8, 1281 (1996).

${ }^{21} \mathrm{M}$. Jupé, L. Jensen, A. Melninkaitis, V. Sirutkaitis, and D. Ristau, Opt. Express 17, 12269 (2009).

${ }^{22}$ S. S. Mao, F. Quéré, S. Guizard, X. Mao, R. E. Russo, G. Petite, and P. Martin, Appl. Phys. A 79, 1695 (2004).

${ }^{23}$ A. P. Pati, I. S. Wahyutama, and A. N. Pfeiffer, Nat. Commun. 6, 7746 (2015).
${ }^{24}$ R. W. Boyd, Nonlinear Optics (Elsevier, 2003).

${ }^{25}$ W. Liu, S. Petit, A. Becker, N. Aközbek, C. M. Bowden, and S. L. Chin, Opt. Commun. 202, 189 (2002).

${ }^{26}$ D. Grojo, A. Mouskeftaras, P. Delaporte, and S. Lei, J. Appl. Phys. 117, 153105 (2015).

${ }^{27}$ M. Lebugle, N. Sanner, N. Varkentina, M. Sentis, and O. Utéza, J. Appl. Phys. 116, 063105 (2014)

${ }^{28}$ J. F. Zhang, S. Taylor, and W. Eccleston, J. Appl. Phys. 72, 1429 (1992).

${ }^{29}$ K. Said, G. Moya, A. Si Ahmed, G. Damamme, and A. Kallel, Appl. Surf. Sci. 361, 226 (2016).

${ }^{30}$ L. Sudrie, A. Couairon, M. Franco, B. Lamoroux, B. Prade, S. Tzortzakis, and A. Mysyrowicz, Phys. Rev. Lett. 89, 186601 (2002).

${ }^{31}$ B. Chimier, O. Utéza, N. Sanner, M. Sentis, T. Itina, P. Lassonde, F. Légaré, F. Vidal, and J. C. Kieffer, Phys. Rev. B 84, 094104 (2011).

${ }^{32}$ B. C. Stuart, M. D. Feit, S. Herman, A. M. Rubenchik, B. W. Shore, and M. D. Perry, Phys. Rev. B 53, 1749 (1996).

${ }^{33}$ M. Mero, J. Liu, W. Rudolph, K. Starke, and D. Ristau, Phys. Rev. B 71, 115109 (2005).

${ }^{34}$ J. R. Gulley, Opt. Eng. 51, 121805 (2012).

${ }^{35}$ L. V. Keldysh, Sov. Phys. JETP 20, 1307 (1965).

${ }^{36}$ S. W. Winkler, I. M. Burakov, R. Stoian, N. M. Bulgakova, A. Husakou, A. Mermillod-Blondin, A. Rosenfeld, D. Ashkenasi, and I. V. Hertel, Appl. Phys. A 84, 413 (2006). 